\title{
Catalytic Asymmetric Ring Opening of meso-Epoxides with Aromatic Amines in Water
}

\author{
Stéphane Azoulay, Kei Manabe, and Shū Kobayashi* \\ Graduate School of Pharmaceutical Sciences, The University of Tokyo, The HFRE Division, \\ ERATO, Japan Science and Technology Agency (JST), Hongo, Bunkyo-ku, Tokyo 113-0033, \\ Japan
}

\section{Supporting Information}

Instrumentation. Proton nuclear magnetic resonance $\left({ }^{1} \mathrm{H}\right.$ NMR) spectra and carbon nuclear magnetic resonance $\left({ }^{13} \mathrm{C}\right.$ NMR) were recorded on a JEOL JNM-LA400 (400 $\left.\mathrm{MHz}\right)$ spectrometer. Chemical shifts for protons and carbons are reported in parts per million (ppm) downfield from tetramethylsilane as an internal standard. Data are presented as follows: chemical shift, multiplicity $(\mathrm{br}=$ broad, $\mathrm{s}=$ singlet, $\mathrm{d}=$ doublet, $\mathrm{t}=$ triplet, $\mathrm{q}=$ quartet, $\mathrm{m}=$ multiplet), coupling constant in Hertz (Hz) and integration. Infrared (IR) spectra were obtained using a JASCO FT/IR-610 spectrophotometer. Data are represented as frequency of absorption $\left(\mathrm{cm}^{-1}\right)$. Optical rotations were measured using a $2 \mathrm{~mL}$ cell with a $1 \mathrm{dm}$ path length on a JASCO P-1010 polarimeter. Data are reported as follows: $[\alpha]_{D}^{t}(\mathrm{c}$ in g per $100 \mathrm{~mL}$, solvent). Melting points were measured on a Yazawa Melting Point BY-I apparatus and are uncorrected. The mass spectroscopic data were obtained on a Bruker Daltonics BioTOF II spectrometer. Chiral HPLC analysis was performed on a Shimadzu VP-series instrument with a chiral stationary phase column as indicated.

Materials. Chiral bipyridine ligand 1 was prepared according to the reported procedure. ${ }^{1}$ Aromatic epoxides $\mathbf{2 d}, \mathbf{2} \mathbf{f}$ and $\mathbf{2 g}$ were prepared by $m$ CPBA oxidation of the corresponding cis-alkenes according to the reported procedure. ${ }^{2}$ All other compounds were commercially available and used as received except aniline and substituted anilines which were distilled from calcium hydride before use. $\mathrm{Sc}(\mathrm{DS})_{3} .3 \mathrm{H}_{2} \mathrm{O}$ was purchased from Wako Chemicals. Preparative thin-layer chromatography (PTLC) was carried out using Wakogel B-5F. The corresponding racemic products were prepared using the achiral scandium-bipyridine complex as catalyst in order to secure the chiral HPLC-assay of the enantioselective reactions.

General Procedure for Catalytic Asymmetric Ring Opening of meso-Epoxides. All asymmetric ring opening reactions were conducted in deionised water under an atmosphere of argon. To a stirred solution of $\mathrm{Sc}(\mathrm{DS})_{3}(0.01$ equiv) in water ( $1 \mathrm{M}$ concentration with respect of the substrates) was added the chiral bipyridine ligand 1 ( 0.012 equiv). The reaction mixture was stirred for one hour at room temperature (rt) upon which the amine and the epoxide were added. Vigorous stirring was continued for 30-48 $\mathrm{h}$ at $\mathrm{rt}$. The reaction was quenched with saturated aqueous $\mathrm{NaHCO}_{3}$. The resultant mixture was extracted with ethyl acetate (three times), and the combined organic layers were dried over anhydrous $\mathrm{Na}_{2} \mathrm{SO}_{4}$. The solvents were evaporated, and the residue was purified by PTLC over silica gel using mixtures of ether-hexane as eluent to give the pure amino alcohol. All known compounds were compared with data reported in the literature; all new compounds were fully

\footnotetext{
${ }^{1}$ Ishikawa, S.; Hamada, T.; Manabe, K.; Kobayashi, S. Synlett 2005, in press.

${ }^{2}$ Lusinchi, X.; Hanquet, G. Tetrahedron 1997, 53, 13727
} 
characterized. The absolute configuration of the products was assigned by comparison with the reported optical rotation values and for the unknown compounds by analogy.

$\mathrm{Ph} \quad{ }_{\mathrm{OH}} \quad(1 S, 2 S)-1,2-D i p h e n y l-2-\left(\right.$ phenylamino)-ethanol (3a). ${ }^{3}$ The title compound was isolated as a white solid; melting point: $100-102{ }^{\circ} \mathrm{C}$. The ee was determined by HPLC using a Daicel Chiralpak AD column (19/1 hexane/ $i$-PrOH; flow rate 1 $\left.\mathrm{mL} / \mathrm{min} ; \tau_{\text {major }}=25.5 \mathrm{~min} ; \tau_{\text {minor }}=29.8 \mathrm{~min}\right)$; ee $=91 \% . \quad[\alpha]_{\mathrm{D}}^{24}=-45.2^{\circ}(\mathrm{c}=$<smiles>[PH2+]c1ccccc1</smiles>
2). IR $\left(\mathrm{cm}^{-1}\right): 3404,3060,1490,1453,1337,1201,1105,1060 .{ }^{\mathbf{1}} \mathbf{H}$ NMR (400 $\mathrm{Hz}, 1 \mathrm{H}), 6.48-6.50(\mathrm{~m}, 2 \mathrm{H}), 6.59-6.6(\mathrm{~m}, 1 \mathrm{H}), 7.0-7.04(\mathrm{~m}, 2 \mathrm{H}), 7.12-7.23(\mathrm{~m}, 10 \mathrm{H}){ }^{13} \mathbf{C}$ NMR $\left(100 \mathrm{MHz}, \mathrm{CDCl}_{3}\right): \delta=64.7,78.0,114.1,117.8,126.7,127.2,127.4,127.8,128.1$, 128.4, 129.0, 140.1, 140.5, 147.2. HRMS (ESI): $[\mathrm{M}+\mathrm{H}]^{+}$calcd. 290.1545, found 290.1537.

$\mathrm{Ph} \quad \mathrm{OH} \quad(1 S, 2 S)-2-\left(N\right.$-Methyl- $N$-phenylamino)-1,2-diphenylethanol (3b). ${ }^{4}$ The title compound was isolated as a white solid; melting point: $57-59{ }^{\circ} \mathrm{C}$. The ee was Ph
$\mathrm{NPh}$
$\mathrm{PrOH}$; flow rate $\left.0.8 \mathrm{~mL} / \mathrm{min} ; \tau_{\text {minor }}=18.2 \mathrm{~min} ; \tau_{\text {major }}=24.3 \mathrm{~min}\right)$; ee $=96 \%$. $[\alpha]^{23}{ }_{\mathrm{D}}=+171.7^{\circ}\left(\mathrm{c}=0.530, \mathrm{CH}_{2} \mathrm{Cl}_{2}\right) . \quad$ IR $\left(\mathrm{cm}^{-1}\right): 3415,3060,3030,2887,1597$, 1499, 1452, 1320, 1190, 754, 698. ${ }^{1} \mathbf{H}$ NMR (400 MHz, $\left.\mathrm{CDCl}_{3}\right): \delta=2.68$ (s, 3H), 3.96 (br s, $1 \mathrm{H}), 4.87(\mathrm{~d}, J=10.1 \mathrm{~Hz}, 1 \mathrm{H}), 5.28(\mathrm{~d}, J=9.6 \mathrm{~Hz}, 1 \mathrm{H}), 6.88-6.92(\mathrm{~m}, 1 \mathrm{H}), 6.96-7.01(\mathrm{~m}, 4 \mathrm{H})$, 7.11-7.29 (m, 8H), $7.38(\mathrm{~d}, J=7.7 \mathrm{~Hz}, 2 \mathrm{H}) .{ }^{13} \mathbf{C ~ N M R}\left(100 \mathrm{MHz}, \mathrm{CDCl}_{3}\right): \delta=32.7,71.5$, $73.7,117.8,120.3,127.6,127.7,127.9,128.2,128.8,129.1,134.6,140.6,151.3$. HRMS (ESI): $[\mathrm{M}+\mathrm{H}]^{+}$calcd. 304.1701, found 304.1691.

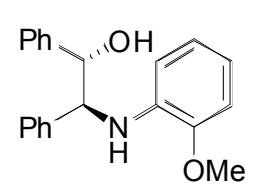

(1S,2S)-2-(2-Methoxyphenylamino)-1,2-diphenylethanol (3c). ${ }^{5}$ The title compound was isolated as a white solid; melting point: $93-95^{\circ} \mathrm{C}$. The ee was determined by HPLC using a Daicel Chiralpak AS-H column (19/1 hexane/ $i$ $\mathrm{PrOH}$; flow rate $\left.0.8 \mathrm{~mL} / \mathrm{min} ; \tau_{\text {major }}=26.9 \mathrm{~min} ; \tau_{\text {minor }}=33.2 \mathrm{~min}\right)$; ee $=93 \%$. $[\alpha]^{24}=-48.0^{\circ}\left(\mathrm{c}=0.540, \mathrm{CH}_{2} \mathrm{Cl}_{2}\right) . \quad$ IR $\left(\mathrm{cm}^{-1}\right): 3398,3061,3028,2934,2857$, $1608,1509,1455,1225,1027,738,701 .{ }^{1} \mathbf{H}$ NMR (400 MHz, $\left.\mathrm{CDCl}_{3}\right): \delta=2.75($ br s, $1 \mathrm{H})$, $3.80(\mathrm{~s}, 3 \mathrm{H}), 4.46(\mathrm{~d}, J=6.4 \mathrm{~Hz}, 1 \mathrm{H}), 4.78(\mathrm{~d}, J=6.4 \mathrm{~Hz}, 1 \mathrm{H}), 6.36(\mathrm{dd}, J=7.8 \mathrm{~Hz}, J=1.4$ $\mathrm{Hz}, 1 \mathrm{H}), 6.58-6.65(\mathrm{~m}, 2 \mathrm{H}), 6.71(\mathrm{dd}, J=7.8 \mathrm{~Hz}, J=1.8 \mathrm{~Hz}, 1 \mathrm{H}), 7.11-7.21(\mathrm{~m}, 10 \mathrm{H}) .{ }^{13} \mathrm{C}$ NMR $\left(100 \mathrm{MHz}, \mathrm{CDCl}_{3}\right): \delta=55.5,64.9,78.2,109.6,111.7,117.1,121.0,126.7,127.3$, 127.7, 128.0, 128.3, 137.1, 140.2, 140.7, 147.4. HRMS (ESI): $[\mathrm{M}+\mathrm{H}]^{+}$calcd. 320.1651, found 320.1638 .

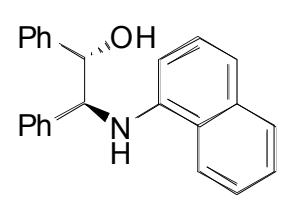

(1S,2S)-2-(Naphthalen-1-ylamino)-1,2-diphenylethanol (3d). The title compound was isolated as a white solid; melting point: $52-55^{\circ} \mathrm{C}$. The ee was determined by HPLC using a Daicel Chiralcel OD column $(19 / 1$ hexane $/ i$-PrOH; flow rate $1 \mathrm{~mL} / \mathrm{min}$; $\tau_{\text {minor }}=24.9 \mathrm{~min} ; \tau_{\text {major }}=50.3 \mathrm{~min}$ ); ee

\footnotetext{
${ }^{3}$ Hou, X.L.; Wu, J.; Dai, L.X.; Xia, L.J.; Tang, M.H. Tetrahedron: Asymmetry. 1998, 9, 1747.

${ }^{4}$ Schneider, C.; Sreekanth, A.R.; Mai, E. Angew. Chem. Int. Ed. 2004, 43, 5691.

${ }^{5}$ Bartoli, G.; Bosco, M.; Carlone, A.; Locatelli, M.; Massaccesi, M; Melchiorre, P. Sambri, L. Org. Lett. 2004, 6, 2173.
} 
$=91 \% \cdot[\alpha]^{22}{ }_{\mathrm{D}}=-144.9^{\circ}\left(\mathrm{c}=0.390, \mathrm{CH}_{2} \mathrm{Cl}_{2}\right) . \quad$ IR $\left(\mathrm{cm}^{-1}\right): 3401,3058,3028,2923,1630,1520$, 1051, 831, 745, 700. ${ }^{1} \mathbf{H}$ NMR $\left(400 \mathrm{MHz}, \mathrm{CDCl}_{3}\right): \delta=2.72($ br s, $1 \mathrm{H}), 4.65(\mathrm{~d}, J=5.5 \mathrm{~Hz}$, $1 \mathrm{H}), 4.91(\mathrm{~d}, J=6.0 \mathrm{~Hz}, 1 \mathrm{H}), 5.51($ br s, $1 \mathrm{H}), 6.26(\mathrm{~d}, J=6.9 \mathrm{~Hz}, 1 \mathrm{H}), 7.05-7.28(\mathrm{~m}, 12 \mathrm{H})$, 7.39-7.47 (m, 2H), $7.73(\mathrm{~d}, J=7.8 \mathrm{~Hz}, 1 \mathrm{H}), 7.96(\mathrm{~d}, J=7.8 \mathrm{~Hz}, 1 \mathrm{H}) .{ }^{13}$ C NMR $(100 \mathrm{MHz}$, $\left.\mathrm{CDCl}_{3}\right): \delta=64.4,78.2,106.6,117.6,120.0,123.9,124.8,125.6,126.4,126.5,127.2,127.5$, $127.9,128.3,128.5,128.6,134.2,139.9,140.7,142.1$. HRMS (ESI): $[\mathrm{M}+\mathrm{H}]^{+}$calcd. 340.1701 , found 340.1698 .

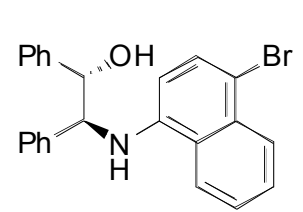

(1S,2S)-2-(4-Bromo-naphthalen-1-ylamino)-1,2-diphenylethanol (3e). The title compound was isolated as a white solid; melting point: $63-65^{\circ} \mathrm{C}$. The ee was determined by HPLC using a Daicel Chiralcel OD column (9/1 hexane $/ i-\mathrm{PrOH}$; flow rate $1 \mathrm{~mL} / \mathrm{min} ; \tau_{\text {minor }}=18.0 \mathrm{~min}$; $\tau_{\text {major }}=24.7 \mathrm{~min}$ ); ee $=86 \% .[\alpha]^{22}=-87.8^{\circ}\left(\mathrm{c}=0.435, \mathrm{CH}_{2} \mathrm{Cl}_{2}\right)$. IR $\left(\mathrm{cm}^{-1}\right): 3423,3063,3009$, 2923, 1590, 1523, 1475,1380, 1052, 752, 700. ${ }^{1} \mathbf{H}$ NMR (400 MHz, $\left.\mathrm{CDCl}_{3}\right): \delta=2.53$ (br s, $1 \mathrm{H}), 4.64(\mathrm{~d}, J=5.5 \mathrm{~Hz}, 1 \mathrm{H}), 4.98(\mathrm{~d}, J=5.0 \mathrm{~Hz}, 1 \mathrm{H}), 5.61(\mathrm{br} \mathrm{s}, 1 \mathrm{H}), 6.1(\mathrm{~d}, J=8.2 \mathrm{~Hz}, 1 \mathrm{H})$, $7.19-7.34(\mathrm{~m}, 11 \mathrm{H}), 7.49-7.57(\mathrm{~m}, 2 \mathrm{H}), 7.97(\mathrm{~d}, J=8.2 \mathrm{~Hz}, 1 \mathrm{H}), 8.15(\mathrm{dd}, J=8.7 \mathrm{~Hz}, J=1.4$ $\mathrm{Hz}, 1 \mathrm{H}) .{ }^{13} \mathbf{C}$ NMR $\left(100 \mathrm{MHz}, \mathrm{CDCl}_{3}\right): \delta=64.3,78.2,107.2,110.3,120.4,125.2,125.5$, $126.4,127.0,127.1,127.7,127.8,128.1,128.4,128.6,130.1,132.1,139.5,140.5,142.1$. HRMS (ESI): $[\mathrm{M}+\mathrm{H}]^{+}$calcd. 418.0807, found 418.0793 .

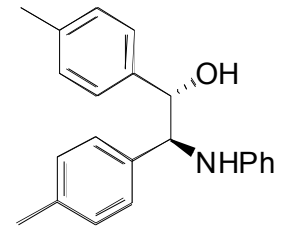

(1S,2S)-2-(phenylamino)-1,2-Di(p-tolyl)-ethanol (3f). The title compound was isolated as a white solid; melting point: $41-43^{\circ} \mathrm{C}$. The ee was determined by HPLC using a Daicel Chiralpak AD-H column (19/1 hexane $/ i$-PrOH; flow rate $1 \mathrm{~mL} / \mathrm{min}$; $\tau_{\text {major }}=26.2 \mathrm{~min} ; \tau_{\text {minor }}=31.8 \mathrm{~min}$ ); ee $=90 \% . \quad[\alpha]^{24}=-47.3^{\circ}\left(\mathrm{c}=0.54, \mathrm{CH}_{2} \mathrm{Cl}_{2}\right) . \quad$ IR $\left(\mathrm{cm}^{-1}\right): 3400,3019,2920$, $1602,1503,1318,1265,1050,820,749,691 .{ }^{1} \mathbf{H} \mathbf{N M R}\left(400 \mathrm{MHz}, \mathrm{CDCl}_{3}\right): \delta=2.24(\mathrm{~s}, 3 \mathrm{H})$, $2.27(\mathrm{~s}, 3 \mathrm{H}), 2.75(\mathrm{br} \mathrm{s}, 1 \mathrm{H}), 4.43(\mathrm{~d}, J=6.0 \mathrm{~Hz}, 1 \mathrm{H}), 4.72(\mathrm{~d}, J=6.0 \mathrm{~Hz}, 1 \mathrm{H}), 6.48(\mathrm{~d}, J=$ $8.2 \mathrm{~Hz}, 2 \mathrm{H}), 6.60(\mathrm{t}, J=7.3 \mathrm{~Hz}, 1 \mathrm{H}), 6.99-7.12(\mathrm{~m}, 10 \mathrm{H}) .{ }^{13} \mathbf{C ~ N M R}\left(100 \mathrm{MHz}, \mathrm{CDCl}_{3}\right): \delta$ $=20.9,21.0,64.2,77.7,114.0,117.6,126.4,127.1,128.8,128.9,129.1,136.8,137.2,137.3$, 137.6, 147.3. HRMS (ESI): $[\mathrm{M}+\mathrm{H}]^{+}$calcd. 318.1878, found 318.1900.

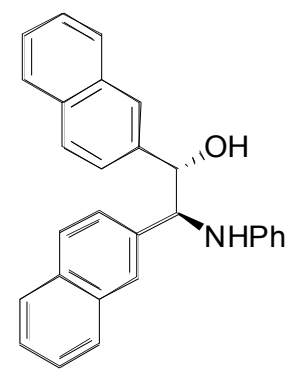

$(1 S, 2 S)-1,2-D i(n a p h t h a l e n-2-y l)-2-p h e n y l a m i n o-e t h a n o l ~(3 g) .{ }^{4}$ The title compound was isolated as a white solid; melting point: $146-148^{\circ} \mathrm{C}$. The ee was determined by HPLC using a Daicel Chiralcel OD column $(9 / 1$ hexane $/ i-\mathrm{PrOH}$; flow rate $1 \mathrm{~mL} / \mathrm{min} ; \tau_{\text {minor }}=38.2 \mathrm{~min} ; \tau_{\text {major }}=53.7 \mathrm{~min}$ ); ee $=91 \% .[\alpha]^{22}{ }_{\mathrm{D}}=-133.8^{\circ}\left(\mathrm{c}=0.410, \mathrm{CH}_{2} \mathrm{Cl}_{2}\right) . \quad$ IR $\left(\mathrm{cm}^{-1}\right): 3399,3052,2923$, $1601,1503,1317,1265,1051,819,749$. ${ }^{1} \mathbf{H}$ NMR (400 MHz, $\left.\mathrm{CDCl}_{3}\right): \delta=$ $2.90($ br s, $1 \mathrm{H}), 4.76(\mathrm{~d}, J=6.0 \mathrm{~Hz}, 1 \mathrm{H}), 5.04(\mathrm{~d}, J=5.5 \mathrm{~Hz}, 1 \mathrm{H}), 6.52(\mathrm{~d}, J$ $=8.7 \mathrm{~Hz}, 2 \mathrm{H}), 6.57-6.61(\mathrm{~m}, 1 \mathrm{H}), 6.98-7.02(\mathrm{~m}, 2 \mathrm{H}), 7.28-7.32(\mathrm{~m}, 2 \mathrm{H})$, 7.37-7.43 (m, 4H), 7.66-7.76 (m, 8H). ${ }^{13} \mathbf{C} \mathbf{N M R}\left(100 \mathrm{MHz}, \mathrm{CDCl}_{3}\right): \delta=64.6,76.7,114.1$, $117.9,124.4,125.3,125.6,125.8,126.0,126.1,126.2,127.9,128.0,128.4,129.0,132.9$, 133.0, 133.1, 133.3, 137.8, 138.0, 147.2. HRMS (ESI): $[\mathrm{M}+\mathrm{H}]^{+}$calcd. 390.1858, found 390.1849 .

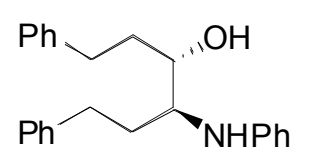

(3S,4S)-1,6-Diphenyl-4-phenylamino-hexan-3-ol (3h). The title compound was isolated as a colorless oil. The ee was determined by HPLC 
using a Daicel Chiralcel OD column $\left(9 / 1\right.$ hexane $/ i-\mathrm{PrOH}$; flow rate $0.8 \mathrm{~mL} / \mathrm{min} ; \tau_{\text {major }}=32.3$ min; $\left.\tau_{\text {minor }}=37.4 \mathrm{~min}\right)$; ee $=60 \% .[\alpha]^{22}{ }_{\mathrm{D}}=-1.7^{\circ}\left(\mathrm{c}=0.525, \mathrm{CH}_{2} \mathrm{Cl}_{2}\right) . \quad$ IR $\left(\mathrm{cm}^{-1}\right): 3047,3050$, 3024, 2926, 2857, 1600, 1497, 1454, 1318, 1060, 748, 698. ${ }^{1} \mathbf{H} \mathbf{N M R}\left(400 \mathrm{MHz}, \mathrm{CDCl}_{3}\right): \delta=$ 1.74-1.86 (m, 3H), 1.90-1.99 (m, 1H), 2.25 (br s, 1H), 2.56-2.73 (m, 3H), 2.77-2.84 (m, 1H), 3.31-3.34 (m, 1H), 3.61-3.65(m, 1H), 5.57-6.60 (m, 2H), $6.70(\mathrm{td}, J=7.3 \mathrm{~Hz}, J=0.9 \mathrm{~Hz}, 1 \mathrm{H})$, 6.71-7.29 (m, 12H). ${ }^{13} \mathbf{C}$ NMR $\left(100 \mathrm{MHz}, \mathrm{CDCl}_{3}\right): \delta=32.2,32.4,34.3,35.8,57.7,72.8$, 117.6, 125.9, 126.0, 128.4, 128.5, 129.4, 141.6, 141.9, 148.3. HRMS (ESI): $[\mathrm{M}+\mathrm{H}]^{+}$calcd. 346.2171 , found 346.2182 .

$n-\mathrm{Bu} \quad \mathrm{OH} \quad(5 S, 6 S)-6$-Phenylamino-decan-5-ol (3i). The title compound was isolated as a colorless oil. The ee was determined by HPLC using a Daicel Chiralpak $n-\mathrm{Bu}$ NHPh AD-H column (19/1 hexane $/ i-\mathrm{PrOH}$; flow rate $0.8 \mathrm{~mL} / \mathrm{min} ; \tau_{\text {minor }}=11.7 \mathrm{~min}$; $\left.\tau_{\text {major }}=12.8 \mathrm{~min}\right)$; ee $=71 \% . \quad[\alpha]^{22}{ }_{\mathrm{D}}=-2.9^{\circ}\left(\mathrm{c}=0.515, \mathrm{CH}_{2} \mathrm{Cl}_{2}\right) . \quad$ IR $\left(\mathrm{cm}^{-1}\right)$ : 3405, 2950, 2951, 2858, 1601, 1457, 747, 692. ${ }^{1} \mathbf{H}$ NMR $\left(400 \mathrm{MHz}, \mathrm{CDCl}_{3}\right): \delta=0.84-0.92$ $(\mathrm{m}, 6 \mathrm{H}), 1.25-1.65(\mathrm{~m}, 12 \mathrm{H}), 3.23-3.28(\mathrm{~m}, 1 \mathrm{H}), 3.56-3.60(\mathrm{~m}, 1 \mathrm{H}), 6.62-6.70(\mathrm{~m}, 3 \mathrm{H}), 7.13-$ $7.24(\mathrm{~m}, 2 \mathrm{H}) .{ }^{13} \mathbf{C} \mathbf{N M R}\left(100 \mathrm{MHz}, \mathrm{CDCl}_{3}\right): \delta=14.0,14.1,22.8,28.2,28.4,32.2,33.8,73.5$, 113.5, 117.4, 129.3, 148.6. HRMS (ESI): $[\mathrm{M}+\mathrm{H}]^{+}$calcd. 250.2171, found 250.2169. 